This item was submitted to Loughborough's Research Repository by the author.

Items in Figshare are protected by copyright, with all rights reserved, unless otherwise indicated.

Strings in charge-transfer Mott insulators: effects of lattice vibrations and the Coulomb interaction

PLEASE CITE THE PUBLISHED VERSION

LICENCE

CC BY-NC-ND 4.0

REPOSITORY RECORD

Alexandrov, A.S., and V.V. Kabanov. 2019. "Strings in Charge-transfer Mott Insulators: Effects of Lattice Vibrations and the Coulomb Interaction”. figshare. https://hdl.handle.net/2134/1207. 


\title{
Strings in charge-transfer Mott insulators: effects of lattice vibrations and the Coulomb interaction
}

\author{
A.S. Alexandrov* and V.V. Kabanov** \\ ${ }^{*}$ Department of Physics, Loughborough University, Loughborough LE11 3TU, United Kingdom, **Josef Stefan Institute 1001, \\ Ljubljana, Slovenia
}

Applying the canonical transformation with the $1 / \lambda$ perturbation expansion in the nonadiabatic and intermediate regime and the discrete generalisation of Pekar's continuous nonlinear equation in the extreme adiabatic regime we show that there are no strings in narrow-band ionic insulators due to the Fröhlich electron-phonon interaction alone. The multipolaron system is a homogeneous state in a wide range of physically interesting parameters, no matter how strong correlations are. At the same time the Fröhlich interaction allows the antiferromagnetic interactions and/or a short-range electron-phonon interactions to form short strings in doped antiferromagnetic insulators if the static dielectric constant is large enough.

The electron-phonon interaction is strong in ionic cuprates and manganites as established both experimentally [1] 4 ] and theoretically [5] 8 . The carriers, doped into the Mott insulator, are coupled with the antiferromagnetic background as well. The antiferromagnetic interactions are thought to give rise to spin and charge segregation (stripes) [9, 10]. There is growing experimental evidence [11 13 that stripes occur in slightly doped insulators. Their theoretical studies were restricted so far to the repulsive strongly correlated models [9, 10, or to an extreme adiabatic limit of the electron-phonon interaction in narrow 14, 15 and wide band 16.17 polar semiconductors and polymers. On the other hand there is strong evidence that the nonadiabatic electronphonon interaction and small polarons are involed in the physics of stripes [3,12]. Also the role of the long-range Coulomb and Fröhlich interactions remains to be properly addressed.

In this letter we prove that the Fröhlich electronphonon interaction combined with the direct Coulomb repulsion does not lead to charge segregation like strings in doped narrow-band insulators, both in the nonadiabatic and adiabatic regimes. However, this interaction significantly reduces the Coulomb repulsion, which might allow much weaker antiferromagnetic and/or short-range electron-phonon interactions to segregate charges in the doped insulators, as suggested by previous studies [9, 10,14$]$.

To begin with, we consider a generic Hamiltonian, including, respectively, the kinetic energy of carriers, the Fröhlich electron-phonon interaction, phonon energy, and the Coulomb repulsion as

$$
H=\sum_{i \neq j} t(\mathbf{m}-\mathbf{n}) \delta_{s, s^{\prime}} c_{i}^{\dagger} c_{j}+\sum_{\mathbf{q}, i} \omega_{\mathbf{q}} n_{i}\left[u_{i}(\mathbf{q}) d_{\mathbf{q}}+H . c .\right]
$$

$$
+\sum_{\mathbf{q}} \omega_{\mathbf{q}}\left(d_{\mathbf{q}}^{\dagger} d_{\mathbf{q}}+1 / 2\right)+\frac{1}{2} \sum_{i \neq j} V(\mathbf{m}-\mathbf{n}) n_{i} n_{j}
$$

with bare hopping integral $t(\mathbf{m})$, and matrix element of the electron-phonon interaction

$$
u_{i}(\mathbf{q})=\frac{1}{\sqrt{2 N}} \gamma(\mathbf{q}) e^{i \mathbf{q} \cdot \mathbf{m}} .
$$

Here $i=(\mathbf{m}, s), j=\left(\mathbf{n}, s^{\prime}\right)$ include site $\mathbf{m}, \mathbf{n}$ and spin $s, s^{\prime}$ quantum numbers, $n_{i}=c_{i}^{\dagger} c_{i}, c_{i}, d_{\mathbf{q}}$ are the electron (hole) and phonon operators, respectively, and $N$ is the number of sites. At large distances ( or small $q$ ) one finds

$$
\gamma(\mathbf{q})^{2} \omega_{\mathbf{q}}=\frac{4 \pi e^{2}}{\kappa q^{2}}
$$

and

$$
V(\mathbf{m}-\mathbf{n})=\frac{e^{2}}{\epsilon_{\infty}|\mathbf{m}-\mathbf{n}|} .
$$

The phonon frequency $\omega_{\mathbf{q}}$ and the static and highfrequency dielectric constants in $\kappa^{-1}=\epsilon_{\infty}^{-1}-\epsilon_{0}^{-1}$ are those of the host insulator $(\hbar=c=1)$.

One can apply the canonical transformation [18] and the $1 / \lambda$ multi-polaron perturbation theory [5] to integrate out phonons,

$$
S=\sum_{\mathbf{q}, i} n_{i}\left[u_{i}(\mathbf{q}) d_{\mathbf{q}}-H . c .\right] .
$$

The result is 5,18

$$
\begin{aligned}
\tilde{H} & =e^{S} H e^{-S}=\sum_{i \neq j} \hat{\sigma}_{i j} c_{i}^{\dagger} c_{j}-E_{p} \sum_{i} n_{i} \\
& +\sum_{\mathbf{q}} \omega_{\mathbf{q}}\left(d_{\mathbf{q}}^{\dagger} d_{\mathbf{q}}+1 / 2\right)+\frac{1}{2} \sum_{i \neq j} v_{i j} n_{i} n_{j},
\end{aligned}
$$

where

$$
\hat{\sigma}_{i j}=t(\mathbf{m}-\mathbf{n}) \delta_{s, s^{\prime}} \exp \left(\sum_{\mathbf{q}}\left[u_{i}(\mathbf{q})-u_{j}(\mathbf{q})\right] d_{\mathbf{q}}-H . c .\right)
$$

is the renormalised hopping integral depending on the phonon variables, $E_{p}=z t \lambda$ is the polaron level shift and

$$
v_{i j}=V(\mathbf{m}-\mathbf{n})-\frac{1}{N} \sum_{\mathbf{q}} \gamma(\mathbf{q})^{2} \omega_{\mathbf{q}} \cos [\mathbf{q} \cdot(\mathbf{m}-\mathbf{n})]
$$


is the net interaction of polarons comprising the longrange Coulomb repulsion and the long-range attraction due to ionic lattice deformations. Here $\lambda=$ $\sum_{\mathbf{q}} \gamma(\mathbf{q})^{2} \omega_{\mathbf{q}} / 2 N z t$ is the dimensionless coupling constant, $t$ is the nearest neighbour hopping integral and $z$ is the coordination lattice number.

The extention of the deformation surrounding (Fröhlich) polarons is large, so their deformation fields overlap at finite density. However, taking into account both the long-range attraction of polarons due to the lattice deformations and the direct Coulomb repulsion, the net long-range interaction is repulsive [5]. At distances larger than the lattice constant, $|\mathbf{m}-\mathbf{n}| \geq a \equiv 1$, this interaction is significantly reduced to

$$
v_{i j}=\frac{e^{2}}{\epsilon_{0}|\mathbf{m}-\mathbf{n}|} .
$$

Optical phonons nearly nullify the bare Coulomb repulsion in ionic solids if $\epsilon_{0}>>1$, which is normally the case in oxides. The kinetic energy term in the exact Hamiltonian, Eq.(6) involves multiphonon events generating a residual polaron-phonon interaction [5]. Below we show that in the two opposite limits, the nonadiabatic $\left(\omega_{\mathbf{q}} \geq t\right)$ and in the extreme adiabatic $\left(\omega_{\mathbf{q}} \rightarrow 0\right)$ regimes, there is no charge segregation or any other instability of the polaronic liquid due to the Fröhlich interaction in doped insulators, but only Wigner crystallization at very low densities.

First we consider the nonadiabtic and intermediate regime. The properties of a single small polaron with the Fröhlich electron-phonon interaction were discussed a long time ago 19,20]. Exact Quantum Monte-Carlo simulations [21] showed that the first order $1 / \lambda$ perturbation theory is numerically accurate for any coupling if the phonon frequency is sufficiently large, $\omega_{\mathbf{q}}>t / 2$. The characteristic frequency of phonons strongly coupled with carriers is about $\omega_{\mathbf{q}}=75 \mathrm{meV}$ [2] in cuprates, so cuprates are in this regime. Hence, one can replace the hopping operator in Eq.(6) for its phonon average, reducing the problem to narrow-band fermions with the weak repulsive interaction, Eq.(9). Next order corrections in $1 / \lambda$ increase the polaron binding energy with little effect on the bandwidth [22. Because the net long-range repulsion is relatively weak, the relevant dimensionless parameter $r_{s}=m^{*} e^{2} / \epsilon_{0}(4 \pi n / 3)^{1 / 3}$ is not very large in doped cuprates. Wigner crystallization appears around $r_{s} \simeq 100$ or larger, which corresponds to the atomic density of polarons $n \leq 10^{-6}$ with $\epsilon_{0}=30$ and the polaronic mass $m^{*}=5 m_{e}$ typical for cuprates and manganites. This estimate shows that small polarons in cuprates and manganites are in the homogeneous state at physically interesting densities.

In the opposite adiabatic limit one can apply a discrete version of the continuos nonlinear equation 23] proposed in Ref. 24] for the Holstein (molecular) model of the electron-phonon interaction and extended to the case of the deformation and Fröhlich interactions in Ref. [14,15. Applying the Hartree approximation for the Coulomb repulsion, the single-particle wave-function, $\psi_{\mathbf{n}}$ (the amplitude of the Wannier state $|\mathbf{n}\rangle$ ) obeys the following equation

$$
-\sum_{\mathbf{m} \neq 0} t(\mathbf{m})\left[\psi_{\mathbf{n}}-\psi_{\mathbf{n}+\mathbf{m}}\right]-e \phi_{\mathbf{n}} \psi_{\mathbf{n}}=E \psi_{\mathbf{n}}
$$

The potential $\phi_{\mathbf{n}, k}$ acting on a fermion $k$ at the site $\mathbf{n}$ is created by the polarization of the lattice $\phi_{\mathbf{n}, k}^{l}$ and by the Coulomb repulsion with the other $M-1$ fermions, $\phi_{\mathbf{n}, k}^{c}$,

$$
\phi_{\mathbf{n}, k}=\phi_{\mathbf{n}, k}^{l}+\phi_{\mathbf{n}, k}^{c} .
$$

Both potentials satisfy the descrete Poisson equation as

$$
\kappa \Delta \phi_{\mathbf{n}, k}^{l}=4 \pi e \sum_{p=1}^{M}\left|\psi_{\mathbf{n}, p}\right|^{2},
$$

and

$$
\epsilon_{\infty} \Delta \phi_{\mathbf{n}, k}^{c}=-4 \pi e \sum_{p=1, p \neq k}^{M}\left|\psi_{\mathbf{n}, p}\right|^{2},
$$

with $\Delta \phi_{\mathbf{n}}=\sum_{\mathbf{m}}\left(\phi_{\mathbf{n}}-\phi_{\mathbf{n}+\mathbf{m}}\right)$. Differently from Ref. [15] we include the Coulomb interaction in Pekar's functional $J$ [23], describing the total energy, in a selfconsistent manner using the Hartree approximation, so that

$$
\begin{aligned}
J & =-\sum_{\mathbf{n}, p, \mathbf{m} \neq 0} \psi_{\mathbf{n}, p}^{*} t(\mathbf{m})\left[\psi_{\mathbf{n}, p}-\psi_{\mathbf{n}+\mathbf{m}, p}\right] \\
& -\frac{2 \pi e^{2}}{\kappa} \sum_{\mathbf{n}, p, \mathbf{m}, q}\left|\psi_{\mathbf{n}, p}\right|^{2} \Delta^{-1}\left|\psi_{\mathbf{m}, q}\right|^{2} \\
& +\frac{2 \pi e^{2}}{\epsilon_{\infty}} \sum_{\mathbf{n}, p, \mathbf{m}, q \neq p}\left|\psi_{\mathbf{n}, p}\right|^{2} \Delta^{-1}\left|\psi_{\mathbf{m}, q}\right|^{2} .
\end{aligned}
$$

If we assume, following Ref. [14 that the single-particle function of a fermion trapped in a string of the length $N$ is a simple exponent, $\psi_{n}=N^{-1 / 2} \exp (i k n)$ with the periodic boundary conditions, then the functional $J$ is expressed as $J=T+U$, where $T=-2 t(N-$ 1) $\sin (\pi M / N) /[N \sin (\pi / N)]$ is the kinetic energy (for an odd number $M$ of spinless fermions) [26], proportional to $t$, and

$$
U=-\frac{e^{2}}{\kappa} M^{2} I_{N}+\frac{e^{2}}{\epsilon_{\infty}} M(M-1) I_{N},
$$

corresponds to the polarisation and the Coulomb energies. Here the integral $I_{N}$ is given by

$$
I_{N}=\frac{\pi}{(2 \pi)^{3}} \int_{-\pi}^{\pi} d x \int_{-\pi}^{\pi} d y \int_{-\pi}^{\pi} d z \frac{\sin (N x / 2)^{2}}{N^{2} \sin (x / 2)^{2}}
$$




$$
\times(3-\cos x-\cos y-\cos z)^{-1} .
$$

It has the following asymptotics, Fig.1,

$$
I_{N}=\frac{1.31+\ln N}{N}
$$

which is also derived analyticially at large $N$ by the use of the fact that $\sin (N x / 2)^{2} /\left(2 \pi N \sin (x / 2)^{2}\right)$ can be replaced by a $\delta$ - function. If we split the first (attractive) term in Eq.(15) into two parts by replacing $M^{2}$ for $M+M(M-1)$, then it becomes clear that the net interaction between polarons remains repulsive in the adiabatic regime as well because $\kappa>\epsilon_{\infty}$. Hence, there are no strings within the Hartree approximation for the Coulomb interaction. Strong correlations do not change this conclusion. Indeed, if we take the Coulomb energy of spinless one-dimensional fermions comprising both Hartree and exchange terms as 25]

$$
E_{C}=\frac{e^{2} M(M-1)}{N \epsilon_{\infty}}[0.916+\ln M]
$$

the polarisation and Coulomb energy per particle becomes (for large $M>>1$ )

$$
U / M=\frac{e^{2} M}{N \epsilon_{\infty}}[0.916+\ln M-\alpha(1.31+\ln N)],
$$

where $\alpha=1-\epsilon_{\infty} / \epsilon_{0}<1$. Minimising this energy with respect to the length of the string $N$ we find

$$
N=M^{1 / \alpha} \exp (-0.31+0.916 / \alpha)
$$

and

$$
(U / M)_{\min }=-\frac{e^{2}}{\kappa} M^{1-1 / \alpha} \exp (0.31-0.916 / \alpha) .
$$

Hence, the potential energy per particle increases with the number of particles so that the energy of $M$ well separated polarons is lower than the energy of polarons trapped in a string no matter correlated or not. The opposite conclusion of Ref. [15] originates in an incorrect approximation of the integral $I_{N} \propto N^{0.15} / N$. The correct asymptotic result is $I_{N}=\ln (N) / N$.

One can argue 27] that a finite kinetic energy $(t)$ can stabilise a string of a finite length. Unfortunately, this is not correct either. We performed exact (numerical) calculations of the total energy $E(M, N)$ of $M$ spinless fermions in a string of the length $N$ including both kinetic and potential energy with the typical values of $\epsilon_{\infty}=5$ and $\epsilon_{0}=30$. The local energy minima (per particle) in the string of the length $1 \leq N \leq 69$ containing $M \leq N / 2$ particles are presented in the Table. Strings with the even fermion numbers carry a finite current and hence the local minima are found for odd $M$. In the extreme wide band regime with $t$ as large as $1 \mathrm{eV}$ the global string energy minimum is found at $M=3, N=25(E=-2.1167$
$\mathrm{eV})$, and at $M=3, N=13$ for $t=0.5 \mathrm{eV}(E=-1.2138$ $\mathrm{eV})$. However, this is not the ground state energy in both cases. The energy of well separated $d \geq 2$-dimensional polarons is well below, less than $-2 d t$ per particle (i.e. $-6 \mathrm{eV}$ in the first case and $-3 \mathrm{eV}$ in the second one in the three dimensional cubic lattice, and $-4 \mathrm{eV}$ and $-2 \mathrm{eV}$, respectively, in the two-dimensional square lattice). This argument is applied for any values of $\epsilon_{0}, \epsilon_{\infty}$ and $t$. As a result we have proved that strings are impossible with the Fröhlich interaction alone contrary to the erroneous Ref. [15,27.

The Fröhlich interaction is, of course, not the only electron-phonon interaction in ionic solids. As discussed in Ref. [5], any short range electron-phonon interaction, like, for example, the Jahn-Teller (JT) distortion can overcome the residual weak repulsion of Fröhlich polarons to form small bipolarons. At large distances small nonadibatic bipolarons weakly repel each other due to the long-range Coulomb interaction, four times of that of polarons, Eq.(9). Hence, they form a liquid state [5], or bipolaronic-polaronic crystal-like structures [28] depending on their effective mass and density. The fact, that the Fröhlich interaction almost nullifies the Coulomb repulsion in oxides justifies the use of the Holstein-Hubbard model [6,29]. The ground state of the 1D Holstein-Hubbard model is a liquid of intersite bipolarons with a significantly reduced mass (compared with the on-site bipolaron) as shown recently [30]. The bound states of three or more polarons are not stable in this model, thus ruling out phase separation. However, the situation might be different if the antiferromagnetic 9, 10, and JT interaction 31] or any short (but finite)-range electron-phonon interaction are strong enough. Due to long-range nature of the Coulomb repulsion the length of a string should be finite (see, also Ref. [12,14]). One can readely estimate its length by the use of Eq.(8) for any type of the short-range electronphonon interaction. If, for example, we take dispersive phonons, $\omega_{\mathbf{q}}=\omega_{0}+\delta \omega\left(\cos q_{x}+\cos q_{y}+\cos q_{z}\right)$ with a $q-$ independent matrix element $\gamma(\mathbf{q})=\gamma$, we obtain a shortrange polaron-polaron attraction as

$$
v_{a t t}(\mathbf{n}-\mathbf{m})=-E_{a t t}(\delta \omega / \omega) \delta_{|\mathbf{n}-\mathbf{m}|, 1},
$$

where $E_{a t t}=\gamma^{2} \omega_{0} / 2$. Taking into account the longrange repulsion as well, Eq.(9), the potential energy of the string with $M=N$ polarons becomes

$$
U=\frac{e^{2}}{\epsilon_{0}} N^{2} I_{N}-\frac{N E_{a t t} \delta \omega}{\omega} .
$$

Minimization of this energy yields the length of the string as

$$
N=\exp \left(\frac{\epsilon_{0} E_{a t t} \delta \omega}{e^{2} \omega}-2.31\right) .
$$

Actually, this expression provides a fair estimate of the string length for any kind of attraction (not only gen- 
erated by phonon dispersion), but also for the antiferromagnetic exchange and/or Jahn-Teller type of interactions 32. Due to the numerical coefficient in the exponent in Eq.(24) one can expect only short strings (if any) with the realistic values of $E_{\text {att }}$ (about a few hundreds millivolts), and the static dielectric constant $\epsilon_{0} \leq 100$.

We conclude that there are no strings in ionic doped insulators with the Fröhlich interaction alone. Depending on their density and mass polarons remain in a liquid state or Wigner crystal. On the other hand the shortrange electron-phonon and/or antiferromagnetic interactions might provide a liquid bipolaronic state and/or charge segregation (strings of a finite length) because the long-range Fröhlich interaction significantly reduces the Coulomb repulsion in highly polarizable ionic insulators.

We greatly appreciate enlightening discussions with Antonio Bianconi, Janez Bonca, Alex Bratkovsky, David Eagles, Pavel Kornilovitch, Fedor Kusmartsev, Dragan Mihailovic, and Jan Zaanen. One of us (V.V.K.) acknowledges support of the work by INTAS grant No.97963.

[1] G. Zhao et al, Nature 385, 236 (1997).

[2] T. Timusk et al, in Anharmonic Properties of High-T Cuprates, eds. D. Mihailović et al, (World Scientific, Singapore, 1995), p.171.

[3] A. Lanzara, et al, Journ. Phys.: Condens. Mat. 11, L541 (1999)

[4] D.R. Temprano et al, Phys. Rev. Lett. 84, 1982 (2000).

[5] A.S. Alexandrov and N.F. Mott, Rep.Prog.Phys. 57, 1197 (1994; 'Polarons and Bipolarons', World Scientific (1995).

[6] A.R. Bishop and M. Salkola , in: 'Polarons and Bipolarons in High- $T_{c}$ Superconductors and Related Materials', eds E.K.H. Salje, A.S. Alexandrov and W.Y. Liang, Cambridge University Press, Cambridge, 353 (1995);

[7] A.J. Millis et al., Phys. Rev. Lett. 74, 5144 (1995);

[8] A.S. Alexandrov and A.M. Bratkovsky, Phys. Rev. Lett. 82, 141 (1999); ibid 84, 2043 (2000).

[9] J. Zaanen and O. Gunnarsson, Phys. Rev. B 40, 7391 (1989).

[10] V. J. Emery et al, Phys. Rev. B 56, 6120 (1997) and refrences therein.

[11] J.M. Tranquada et al, Nature 375, 561 (1996).

[12] A. Bianconi, J. Phys. IV France, 9, 325 (1999) and references therein.

[13] V. J. Emery et al, to appear in Proc. Natl. Acad. Sci USA, preprint cond-mat/9907228.

[14] F.V. Kusmartsev, J. Phys. IV France, 9, 321 (1999).

[15] F.V. Kusmartsev, Phys. Rev. Lett., 84, 530 (2000).

[16] L.N. Grigorov, Makromol. Chem., Macromol. Symp. 37, 159 (1990).

[17] L.N. Grigorov et al, Makromol. Chem., Macromol. Symp. 37, 177 (1990).
TABLE I. $E(M, N)$ for $t=1 \mathrm{eV}$ and $t=0.5 \mathrm{eV}$

\begin{tabular}{lllll}
\hline \hline & $t=1 \mathrm{eV}$ & & $t=0.5 \mathrm{eV}$ & \\
\hline $\mathrm{M}$ & $\mathrm{N}$ & $\mathrm{E}(\mathrm{M}, \mathrm{N})$ & $\mathrm{N}$ & $\mathrm{E}(\mathrm{M}, \mathrm{N})$ \\
\hline 1 & 11 & -2.0328 & 3 & -1.1919 \\
3 & 25 & -2.1167 & 13 & -1.2138 \\
5 & 42 & -2.1166 & 25 & -1.1840 \\
7 & 61 & -2.1127 & 40 & -1.1661 \\
\hline \hline
\end{tabular}

[18] I.G. Lang and Yu.A. Firsov, Zh. Eksp. Teor. Fiz. 43, 1843 (1962) ( Sov. Phys. JETP 16, 1301 (1963)).

[19] J. Yamashita and T. Kurosawa, J. Phys. Chem. Solids 5, 34 (1958).

[20] D.M. Eagles, Phys. Rev. 181, 1278 (1969) and references therein.

[21] A.S. Alexandrov and P.E. Kornilovich, Phys. Rev. Lett. 82, 807 (1999).

[22] Yu.A. Firsov et al, Phys. Rev. B 59, 12132 (1999).

[23] S.I. Pekar, Zh. Eksp. Teor. Fiz. 16, 335 (1946).

[24] V.V. Kabanov and O.Yu. Mashtakov, Phys. Rev. B47, 6060 (1993).

[25] This expression differs from Ref. 14, 15, by the numerical coefficients.

[26] For an even $M$ the kinetic energy is $T=-2 t(N-$ 1) $\sin (\pi M / N) \cos (\pi / N) /[N \sin (\pi / N)]$.

[27] F.V. Kusmartsev, Phys. Rev. Lett. Erratum, 84, 5026 (2000).

[28] S. Aubry, in: 'Polarons and Bipolarons in High- $T_{c}$ Superconductors and Related Materials', eds E.K.H. Salje, A.S. Alexandrov and W.Y. Liang, Cambridge University Press, Cambridge, 271 (1995).

[29] H. Fehske et al, Phys. Rev. B51, 16582 (1995).

[30] J. Bonca et al, Phys. Rev. Lett., 84, (2000).

[31] L.P. Gor'kov and A.V. Sokol, Pis'ma Zh. Eksp. Teor. Fiz., 46, 333 (1987).

[32] $E_{a t t} \delta \omega / \omega \propto J$ in the case of the antiferomagnetic interaction $J$, and $E_{a t t} \delta \omega / \omega \propto E_{J T}$ in the case of the JT distortion ( $E_{J T}$ is the Jahn-Teller energy).

\section{Figure captions}

Fig.1. The polarisation energy of small Fröhlich polarons trapped in a string depends on its length as $\ln (N) / N$. 
arXiv:cond-mat/0005419 v2 5 Dec 2000

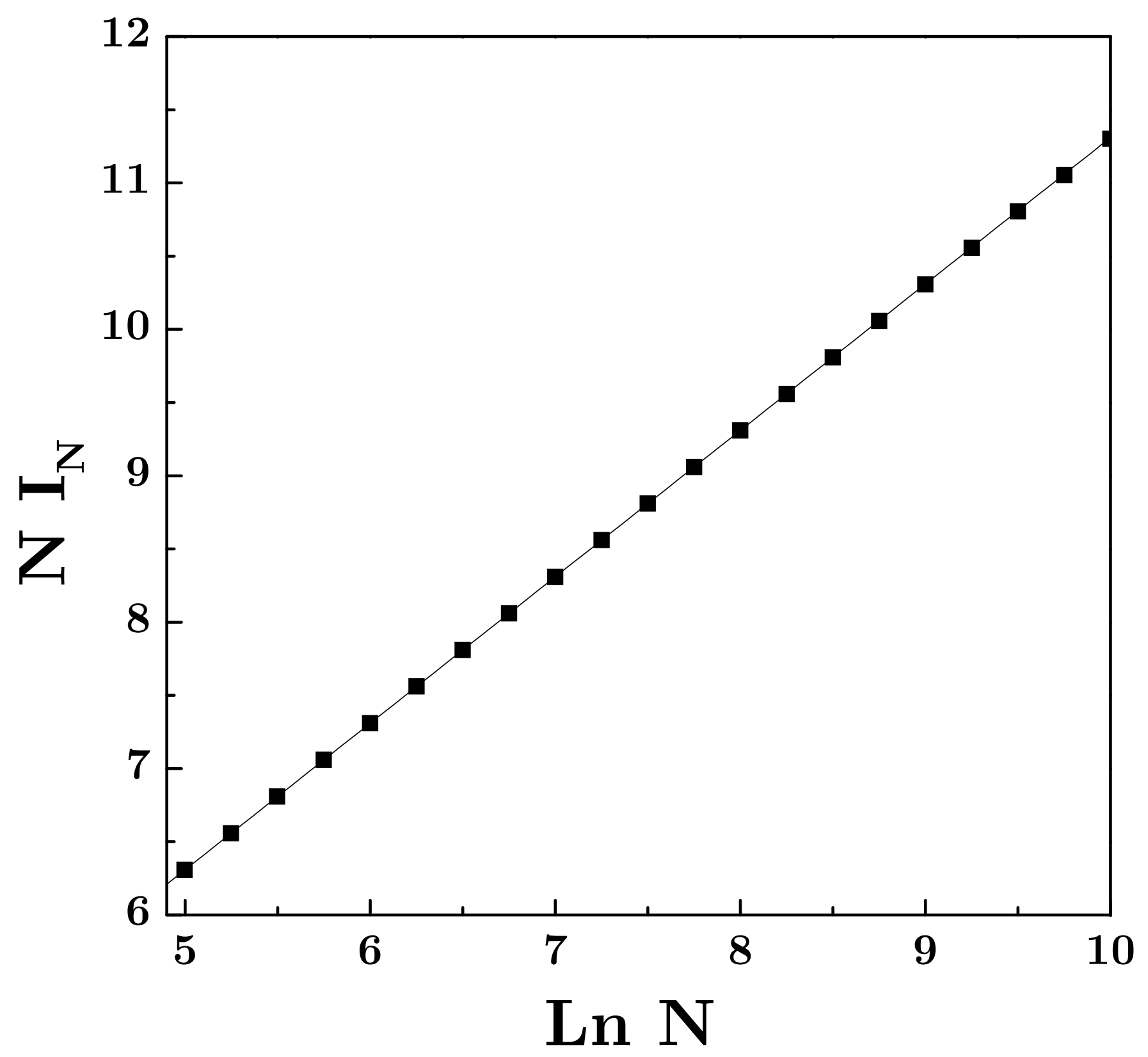

